INPLASY

PROTOCOL

To cite: Keng et al. Influence of Protein Diet on Body Weight Changes in Overweight and

Obese Individuals - A

Systematic Review of

Randomized Controlled Trials

Protocol. Inplasy protocol

202190080. doi:

10.37766/inplasy2021.9.0080

Received: 23 September 2021

Published: 23 September 2021

Corresponding author:

Justin Gnanou

justingnanou@gmail.com

Author Affiliation:

International Medical

University, Malaysia.

Support: Research fund.

Review Stage at time of this submission: Completed but not published.

Conflicts of interest:

None declared.

\section{Influence of Protein Diet on Body Weight Changes in Overweight and Obese Individuals - A Systematic Review of Randomized Controlled Trials Protocol}

Keng, SY1; Caszo, BA²; Che Roos, NA33; Gnanou, JV4.

Review question / Objective: This systematic review was performed to ascertain the optimum amount of protein intake which could lead to weight loss and satiety among overweight and obese individuals.

Condition being studied: We explored the effect of different dietary protein composition on weight loss in overweight and/ or obese individuals.

Information sources: A comprehensive search was performed using electronic bibliographic databases including MEDLINE, Scopus, Cochrane, and PubMed. The search terms used were as follows: "protein diet" AND ("weight change" OR " weight loss" OR "weight reduction" OR "changes in body composition") AND ("obesity" OR "overweight" OR "fat distribution" OR "body fat mass" OR "fat free mass" OR "body mass index") AND ("satiety" OR "fullness" OR "frequency of eating" OR "hunger suppression." References of the included studies were also manually screened for eligible studies. No restrictions on language or publication date was applied. The respective authors were contacted for missing information if necessary.

INPLASY registration number: This protocol was registered with the International Platform of Registered Systematic Review and Meta-Analysis Protocols (INPLASY) on 23 September 2021 and was last updated on 23 September 2021 (registration number INPLASY202190080).

\section{INTRODUCTION}

Review question / Objective: This systematic review was performed to ascertain the optimum amount of protein intake which could lead to weight loss and satiety among overweight and obese individuals.
Rationale: Obesity, in general, is an imbalance between energy intake and expenditure as a result of changes in eating behaviour due to mechanisms related to appetite and satiety. Therefore, change in weight is often associated with variation or modification in diet. Controlled energy 
intake in association with a moderately elevated protein intake may represent an effective and practical weight-loss strategy. It is well established that under most conditions, protein is more satiating than the isoenergetic ingestion of carbohydrate or fat. Hence, a modest increase in protein, at the expense of the other macronutrients, can promote satiety and facilitate weight loss through reduced energy consumption.

Condition being studied: We explored the effect of different dietary protein composition on weight loss in overweight and/or obese individuals.

\section{METHODS}

Search strategy: A comprehensive search was performed using electronic bibliographic databases including MEDLINE, Scopus, Cochrane, and PubMed. The search terms used were as follows: "protein diet" AND ("weight change" OR " weight loss" OR "weight reduction" OR "changes in body composition") AND ("obesity" OR "overweight" OR "fat distribution" OR "body fat mass" OR "fat free mass" OR "body mass index") AND ("satiety" OR "fullness" OR "frequency of eating" OR "hunger suppression." References of the included studies were also manually screened for eligible studies. No restrictions on language or publication date was applied.

Participant or population: Overweight and/ or obese adults of any sex or race.

Intervention: High protein diet for at least 3 weeks duration.

Comparator: Low or normal protein intake for at least 3 weeks duration

Study designs to be included: Randomized controlled trials (RCT).

Eligibility criteria: Studies investigating the effects of dietary protein in adult human who were overweight and/or obese were eligible for inclusion. The intervention must consist of dietary protein from meat, dairy products, nuts, or certain grains and beans.
The protein composition must be at least $20-30 \%$ of the total dietary composition. The intervention can be given as a main meal (e.g. breakfast),snack or supplement. For the intervention group, a dietary protein with a higher composition were considered as the study intervention. The comparator include low or normal protein intake. Only randomized controlled trials (RCT) were considered. Exclusion criteria include:(1) Population (i) Subjects with a genetic disease, Cushing syndrome, and hyperinsulinaemia; (ii) Subjects who have undergone a surgical procedure such as bariatric surgery; (iii) Pregnant subjects; (2) Intervention and comparator used were different than the protocol stated in the inclusion criteria; (3) study duration less than 3 weeks and study design other than RCT (observational studies, reviews, experimental, editorial etc); (4) abstract, conference proceeding; (5) studies with insufficient data/information and failed to report the outcome of interest.

Information sources: A comprehensive search was performed using electronic bibliographic databases including MEDLINE, Scopus, Cochrane, and PubMed. The search terms used were as follows: "protein diet" AND ("weight change" OR " weight loss" OR "weight reduction" OR "changes in body composition") AND ("obesity" OR "overweight" OR "fat distribution" OR "body fat mass" OR "fat free mass" OR "body mass index") AND ("satiety" OR "fullness" OR "frequency of eating" OR "hunger suppression." References of the included studies were also manually screened for eligible studies. No restrictions on language or publication date was applied. The respective authors were contacted for missing information if necessary.

Main outcome(s): 1. Weight loss/changes observed measure in kilogram (kg) or gram (g) reported as mean or percentage. 2. Satiety measured using visual analog scale (VAS), satiety quotient (SQ) or other validated method. 
Additional outcome(s): 1. BMI changes reported as mean or percentage. 2. Energy expenditure.

Data management: Titles and/or abstracts of studies retrieved through the search strategy and those from additional sources were screened independently by two reviewers (SY and BC) to identify studies that potentially meet the inclusion criteria outlined above. The full text of these potentially eligible studies were retrieved and independently assessed for eligibility by the same two review team members. Any disagreement were resolved through discussion with the third reviewer (JG). A standardized, pre-piloted MS Excel form were used to extract data from the included studies for assessment of study quality and evidence synthesis. Extracted information include: author,year/country, study design, sample (n;sex; age), comorbidity, period of intervention, BMI kg/ $\mathrm{m} 2$ (mean/range) of the sample, appetite/ satiety, dietary protocol (intervention/ control), protein source, weight and BMI changes. Two review authors extracted data independently (SY and BC). Any discrepancies identified were resolved through discussion with a third author where necessary (JG). The other authors (JG and NA) checked the final data extraction. Missing data were requested from study authors if necessary.

Quality assessment / Risk of bias analysis: The Cochrane Risk of Bias Tool (ROB 2.0) was used to assess the included studies risk of bias. Two reviewers (SY and NA) independently assessed the quality of each study. A third reviewer (BC) was consulted for disagreements between the two main reviewers. The tool consisted of 6 domains which recommend the reporting of the randomization process, blinding of participants/personnel/assessor, completeness and selectiveness of outcome reporting. Each domain will be judged as at being a "high", "low" or "unclear" risk of bias. If all the domains were answered "low", the risk of bias were considered to be be low and if any item was classified as "high," a high risk of bias will be expected. A scoring system will be designed according to the selected domain which was deemed to be important on a typical type of study. These ratings, however, will not be used as a criterion for study eligibility.

Strategy of data synthesis: A narrative synthesis was planned. Both study characteristics and outcome data were tabulated. The primary outcomes include measurement of association between high protein versus low protein intake and weight changes. In addition, we also identified data satiety and energy expenditure where applicable in relation to the demographic and regional characteristics of the studies. A metaanalysis will be conducted if the data permits. The risk of bias of included studies were summarized and reported narratively.

Subgroup analysis: Where possible, we planned to evaluate the effect of different source of dietary protein and type of meal subgroups.

Sensitivity analysis: A sensitivity analysis will only be conducted if a meta-analysis was performed.

Language: No language restrictions was applied.

Country(ies) involved: Malaysia.

Keywords: Protein diet; weight loss; obesity; overweight; satiety; systematic review.

Dissemination plans: A paper will be submitted to a leading journal in this field.

Contributions of each author:

Author 1 - Sue Yin Keng.

Email: kengsueyin@yahoo.com

Author 2 - Brinnell Caszo.

Email: brinnellcaszo@imu.edu.my

Author 3 - Nur Aishah Che Roos.

Email: nuraishah@upnm.edu.my

Author 4 - Justin Gnanou.

Email: justinvijay@imu.edu.my 